\title{
Kidney Carcinoma
}

National Cancer Institute

\section{Source}

National Cancer Institute. Kidney Carcinoma. NCI Thesaurus. Code C9384.

A carcinoma arising from the epithelium of the renal parenchyma or the renal pelvis. The majority are renal cell carcinomas. Kidney carcinomas usually affect middle aged and elderly adults. Hematuria, abdominal pain, and a palpable mass are common symptoms. 